\title{
Variability of behavioral chronotypes of 16 mammalian species under controlled conditions
}

Roberto Refinetti ${ }^{\mathrm{a},{ }^{*}}$, Thomas Wassmer ${ }^{\mathrm{b}}$, Priyoneel Basu ${ }^{\mathrm{c}}$, Rajeev Cherukalady ${ }^{\mathrm{c}}$, Varun Kumar Pandey $^{\mathrm{c}}$, Muniyandi Singaravel ${ }^{\mathrm{c}}$, Claudia Giannetto $^{\mathrm{d}}$, Giuseppe Piccione ${ }^{\mathrm{d}}$

${ }^{a}$ Circadian Rhythm Laboratory, Department of Psychology, Boise State University, Boise, ID

83725, USA; ${ }^{b}$ Biology Department, Siena Heights University, Adrian, MI 49221, USA;

${ }^{c}$ Department of Zoology, Centre of Advanced Study, Banaras Hindu University, Varanasi

221005, India; ${ }^{d}$ Laboratorio di Cronofisiologia Veterinaria, Dipartimento di Scienze

Veterinarie, Università degli Studi di Messina, 98168 Messina, Italy

* Corresponding author. Tel.: +1-208-426-4117. Fax: +1-208-426-4386.

E-mail address: refinetti@circadian.org (R. Refinetti). 


\begin{abstract}
Human chronotypes (differences in preference for early or late rising each day) have been extensively studied in recent years, but no attempt has been made to compare human chronotypes with the chronotypes of other animal species. We evaluated behavioral chronotypes in 16 mammalian species along a body size gradient of five orders of magnitude (from mice to cattle). Individuals of all species were studied under a 12L:12D photoperiod in a thermoneutral environment with food and water available at all times. Rhythms of locomotor activity were analyzed for onset time, acrophase, and robustness. Neither of these rhythmic parameters was significantly related to body size, but onset time and acrophase varied considerably from species to species, thus characterizing diurnal and nocturnal species. Chronotype spreads ranged from less than an hour in sheep to almost 24 hours in cats, thus extending both below and above the human chronotype spread of 6 hours. The variability of chronotype (as quantified by the standard deviation of group means) was much larger between species than within species and also larger between individuals of a species than within individuals on consecutive days. These results help situate the matter of human chronotypes within the broader context of variability in the phase angle of entrainment of circadian rhythms in animals.
\end{abstract}

Keywords: chronotype, circadian rhythm, locomotor activity, intrasubject variability, intersubject variability 


\section{Introduction}

Forty years ago, Horne and Östberg developed a questionnaire to rapidly classify people along a continuum from "morning types" to "evening types" [1]. Although not explicitly acknowledged by the authors, this classification reflects differences in the phase angle of entrainment of the circadian system of an individual in reference to the environmental cycle of light and darkness. Animals (including humans) have an endogenous pacemaker that generates circadian rhythmicity but that is modulated by environmental stimuli, particularly the light-dark cycle. Given the natural speed of the pacemaker, the speed of the entraining environmental cycle, and the species-specific sensitivity of the pacemaker to the environmental stimulus, the oscillatory pattern of the pacemaker establishes a predictable temporal relationship with the environmental cycle that is called the "phase angle of entrainment" $[2,3]$. The phase angle of entrainment can be defined in reference to any stage of the environmental cycle, although it is often defined in reference to either lights-on (sunrise) or lights-off (sunset). "Morning types" tend to wake up and be more productive early in the day (and, therefore, have an advanced phase angle of entrainment), whereas "evening types" tend to wake up later and be more productive in the afternoon and evening (and, therefore, have a delayed phase angle of entrainment).

The behavioral typology of morningness and eveningness in humans is consistent with the timing of the rhythms of body temperature $[4,5]$, heart rate $[6,7]$, melatonin secretion $[8,9]$, and other physiological variables. Perhaps because significant correlations have been found between morningness-eveningness and several psychological traits [10-14], the classification of chronotypes has attracted great attention from social scientists. As of February 2016, Horne and Östberg's original publication was the article with the highest number of citations of any article retrieved by the keyword "circadian" in the Web of Science database (produced by Thomson Reuters, New York, NY). 
Because differences in chronotypes are reflections of differences in phase angles of entrainment, they are expected to be present not only in humans but in all animal species. Yet, despite occasional observations of variation in the phase angles of entrainment of individual members of a few species [15-19], no comparative study of chronotypes has been conducted. Thousands of studies of the daily rhythm of locomotor activity of individual species have been conducted in the wild and in the laboratory [20,21], but — because of large differences in photoperiod, ambient temperature, food availability, presence of predators, and so on comparisons between different species cannot be reliably conducted in a retrospective manner. In the present study, we compared the activity rhythms of individuals of 16 different mammalian species, ranging in size from mice to cattle, while attempting to provide similar environmental conditions for all animals. We analyzed differences in chronotype not only between species but also within and between different individuals of each species in order to quantify the extent of intra- and intersubject variability of behavioral chronotype.

\section{Materials and method}

\subsection{Subjects}

Animals of 16 different species served as subjects. The names and numbers of individuals of each species are listed in Table 1 in ascending order of body size. The smallest species (Indian field mouse) weighs $13 \mathrm{~g}$, whereas the largest species (cow) weighs $700 \mathrm{~kg}$, thus yielding a body size gradient of five orders of magnitude. All individuals were non-pregnant young adults. Because the locomotor activity rhythm of female rodents is often modulated by the estrous cycle (with earlier activity onsets on the day of ovulation) [20,21], only males were used in rodent species. The rabbits and cats were also all male. Horses and dogs were 50\% male and 50\% female. Cows and sheep were all female. 
The farm animals (cow, horse, and sheep) were procured from and studied at the School of Veterinary Medicine of the University of Messina, in Sicily, Italy. The domestic animals (dog, cat, and rabbit) were also procured from and studied at the University of Messina. Two of the wild animals (palm squirrel and field mouse) were trapped in the surroundings of Varanasi, in Uttar Pradesh, India, and studied at Banaras Hindu University after quarantine and adaptation to captivity. Fox squirrels were captured, fitted with a recording device, and studied in a restricted outdoor area under mild weather on the campus of Siena Heights University (Adrian, Michigan). Animals of the remaining species were either purchased from Charles River Laboratories (Wilmington, Massachusetts) or bred at a regional campus of the University of South Carolina (Walterboro, South Carolina) and studied at this latter location.

\subsection{Procedure}

Experiments were conducted in accordance with the regulations of the Guide for the Care and Use of Laboratory Animals (U.S. National Research Council, 2011), the Guidelines for the Use of Wild Mammals in Research (American Society of Mammalogists, 2011), and European Union's Directive 86/609 CEE.

Because of the large difference in body sizes of the various species, it was not possible to use the exact same procedures of animal husbandry and methods of environmental control and activity monitoring in all species. Nonetheless, efforts were made to have experimental conditions as similar as possible for all species. Either by automated control of lighting and ambient temperature in the laboratory or by selection of the appropriate time of the year in barns, animals of all species were exposed to a light-dark cycle with 12 hours of light and 12 hours of darkness per day (12L:12D) and an ambient temperature of $20-24{ }^{\circ} \mathrm{C}$ (with a daily oscillation of less than $4{ }^{\circ} \mathrm{C}$ ). Food and water were freely available at all times for all species. Fox squirrels were free-ranging outdoors, so that ad libitum availability of food and water could not be strictly 
assured, but maintenance (or increase) of body weight between the beginning and end of data recording indicated that the animals were not in negative energy balance.

Farm animals were housed in individual indoor stalls and were fed hay, oats, corn, and barley in abundance (replenished twice a day at 08:00 and 16:00 hours). Because food was available in abundance, it is unlikely that food replenishment had an effect on the circadian system comparable to the effect of restricted feeding regimes on the circadian system of laboratory rodents. Importantly, this feeding regime is a common regime for farm animals and reflects standard practice in livestock management. Water was available ad libitum. Locomotor activity was recorded with an activity data-logger (Actiwatch, Mini Mitter Co., Bend, OR) strapped to the animal's neck.

Dogs and cats were housed in individual pens $(140$ x $200 \mathrm{~cm})$ and were fed a certified dog/cat diet replenished daily at 09:00 hours. We have previously shown that the wave forms of the daily rhythms of body temperature, heart rate, and blood pressure of the dog are not affected by the time of feeding [22]. Water was available ad libitum. Locomotor activity was recorded with an activity data-logger (Actiwatch, Mini Mitter Co., Bend, OR) strapped to the animal's neck.

Rabbits were individually housed in metallic cages $(90 \mathrm{~cm}$ x $50 \mathrm{~cm}$ x $35 \mathrm{~cm}$ ) and were fed rabbit pellets and water ad libitum. Locomotor activity was recorded with an activity datalogger (Actiwatch, Mini Mitter Co., Bend, OR) strapped to the animal's neck.

Free-ranging fox squirrels were equipped with temperature-sensitive data-logger collars fitted with iButtons (DS1922L, Maxim Integrated Products, San Jose, CA). Because of changes in ambient temperature related to microclimate variations as the animal moves around, the monitoring of collar temperature provides an index of locomotor activity similar to that obtained by the monitoring of differences in the strength of telemetry signals. 
Field mice and palm squirrels were housed in individual housing chambers $(36 \mathrm{~cm} \times 20$ $\mathrm{cm} \times 14 \mathrm{~cm}$ ) lined with wood shavings and were fed commercial rodent pellets and water ad libitum. Locomotor activity was recorded with running wheels using the Clocklab setup for rodent locomotor activity rhythm recording (Coulbourn Instruments, Whitehall, PA).

Domestic mice, rats, gerbils, Syrian hamsters, Siberian hamsters, degus, and Nile grass rats were housed individually in polypropylene cages $(36 \mathrm{~cm}$ x $24 \mathrm{~cm}$ x $19 \mathrm{~cm})$ lined with wood shavings and were fed Purina rodent chow and water ad libitum. A metallic running wheel (18 $\mathrm{cm}$ diameter) was attached to each animal cage. Magnetic switches attached to the running wheels were connected to data acquisition boards (Digital Input Card AR-B2001, Acrosser Technology, Taiwan) linked to desktop computers.

\subsection{Data analysis}

Data were stored and analyzed in 5-min, 6-min, or 10-min bins, depending on the type of device used for the recording of locomotor activity. To avoid sampling bias, animals were not pre-screened for the quality of their locomotor activity rhythms. However, because weak daily rhythmicity due to animal idiosyncrasy cannot be reliably distinguished from weak rhythmicity due to illness or to equipment malfunction, all data sets were initially subjected to a test of daily rhythmicity. If the data set did not exhibit significant rhythmicity between 23.5 and 24.5 hours, as determined both by the Lomb-Scargle periodogram procedure [23] and by cosinor rhythmometry [24], the set was excluded. This occurred very rarely ( 3 individuals total), and the sample sizes given in Table 1 do not include these disqualified animals.

An interval of 10 consecutive days was analyzed for each individual of each species. For descriptive purposes, daily wave-form plots of activity were generated for each species by averaging, time-bin by time-bin, the 10 consecutive days for each animal and then averaging all animals in each species while preserving the temporal resolution of the original data sets. 
For each individual of each species, three parameters of the activity rhythm were analyzed: onset time, acrophase, and robustness. Onset time (the time of initiation of running activity each day) is a classic measure of rhythm phase in circadian biology. In the present study, onset time was determined by a computer algorithm. First, the time series was smoothed by a 7hour moving-averages procedure and phase-advanced by 3.5 hours to correct for the 3.5 -hour phase-delay caused by the moving-averages procedure. Then, for each 24-hour interval, the onset time was computed as the time when the activity level rose above the daily mean. Occasionally (i.e., in fewer than $3 \%$ of the data sets), the algorithm failed to identify an onset for a given day. In these cases, the missing value was replaced with a random number within the range of the remaining onsets.

The second parameter of the activity rhythm to be analyzed was the acrophase (the time of the daily peak of the activity rhythm). The acrophase was computed by the single cosinor procedure, which fits a cosine wave to the data in order to overcome differences in wave form of the time series under analysis [24]. This is particularly important when different species are being compared, as the wave form of the activity rhythm is known to vary greatly from species to species. The acrophase computed by the cosinor procedure provides a "center-of-gravity" measure of the wave form that minimizes the effects of differences in slope, bimodality, etc.

The third parameter to be analyzed was rhythm robustness. Rhythm robustness refers to the strength of rhythmicity and is closely related to the stationarity of the time series [25]. Robustness is independent of amplitude, except at the extreme low end of the range, as a rhythm with zero amplitude also has zero robustness. Rhythm robustness was computed as the percentage of total variance accounted for by the cosine fit [25]. Onset time and acrophase were calculated in local time and expressed in clock hours adjusted to lights-on at 07:00 hours and lights-off at 19:00 hours. 
The spread of individual chronotypes in each of the species was computed as the interval containing $95 \%$ of the individual chronotypes, which is approximately the interval that goes from two standard deviations below the species mean to two standard deviations above the species mean. Intrasubject variability was computed as the standard deviation of the mean of the onsets over 10 days (calculated for each individual of a species and then averaged over all the individuals). Intersubject variability was computed as the standard deviation of the mean of the onsets over all individuals of a species (calculated for each of the 10 days and then averaged over the 10 days).

The statistical significance of differences between group means was evaluated by analysis of variance (ANOVA) followed by post-hoc pairwise comparisons with Tukey's HSD test [26]. The level of significance $(\alpha=0.05)$ was maintained at each ANOVA. Correlation coefficients were computed by the principle of least squares.

For comparative purposes, human data were taken from the study by Roenneberg and colleagues [27], which involved more than 55,000 participants, mostly from Germany, in an online survey of chronotypes. The mean activity onset (wake-up time on free days) for this group was 08:45 hours, with a standard deviation of 90 minutes. Natural variation in photoperiod was not controlled in Roenneberg's study, and we assumed a photophase starting at 07:00 hours and ending at 19:00 hours, which correspond to the times of sunrise and sunset in Berlin in late September (http://www.timeanddate.com). Actual activity data, which were not collected in the study, would be required for the computation of acrophases. In the absence of data, we assumed that the human acrophase occurs in the middle of the active phase of the daily activity-rest cycle (i.e., 8 hours after wake-up time, or 16:45 hours). The assumption of human acrophase occurring in the middle of the active phase is consistent with actigraphic data from a sample of 500 
individuals in the United States [28]. From the latter study, we obtained also an estimate of rhythm robustness.

\section{Results}

Figure 1 shows the average daily activity rhythms for all 16 species. The curves in each panel were generated by averaging, time-bin by time-bin, the data from 10 consecutive days for each animal and then averaging the data for all animals in each species. Inspection of the figure indicates that whereas some species (such as cow and sheep) exhibit several daily clusters of activity other species exhibit unimodal activity patterns with activity concentrated in the light phase of the light-dark cycle (such as horse and fox squirrel) or in the dark phase of the lightdark cycle (such as Syrian hamster and house mouse). Particularly noteworthy is the similarity of the activity patterns of the two species of the genus Mus (house mouse and field mouse).

Figure 2 (top panel) shows the means ( \pm standard errors) of the activity onset times for the 16 species in this study and for human subjects in previous studies [27, 28]. With some interspecies variability, the onset times are clustered around the time of lights-on (07:00 hours) and around the time of lights-off (19:00 hours), thus characterizing a group of diurnal species and a group of nocturnal species. The dispersion of onset times shows no relationship with body size $(r=-0.248, n=17, p=0.661)$. As expected, the acrophases lag behind the onsets by several hours (middle panel), but the distinction between diurnal and nocturnal species is much less clear in the acrophases than in the onsets, with greater variability of acrophases being particularly noticeable among diurnal animals. The bottom panel of Fig. 2 shows rhythm robustness as a function of body size. Although robustness varies from species to species, with maximal robustness in the field mouse and minimal robustness in humans, the correlation with body size does not reach statistical significance $(r=-0.181, n=17, p=0.506)$. 
Figure 3 shows the spread of individual chronotypes in each of the species. The spread was computed as the interval containing $95 \%$ of the individual chronotypes. The spread is as narrow as 40 minutes in sheep and as wide as 23 hours in cats. This means that individual sheep initiate activity each day within a 40 minute window around the species mean, whereas individual cats initiate activity at very different times of the day. It can be noticed that the human chronotype spread of 6 hours is comparable to that of the laboratory rat, is wider than those of seven of the species, and is narrower than those of nine of the species. Because greater variability of onsets from individual to individual might be a computational artifact resulting from weak rhythmicity, we computed a correlation coefficient between chronotype spread and rhythm robustness. We found $r=-0.439, n=17, p=0.075$, which does not substantiate the suspicion of significantly wider chronotype spreads in species with weaker rhythmicity.

Figure 4 shows the indices of intrasubject and intersubject variabilities for the 16 species in this study. Intrasubject variability refers to how variable the activity pattern of an individual is from day to day and was computed as the standard deviation of the mean of the onsets over 10 days (calculated for each individual of a species and then averaged over all the individuals). Intersubject variability refers to how variable the activity patterns of different individuals are and was computed as the standard deviation of the mean of the onsets over all individuals of a species (calculated for each of the 10 days and then averaged over the 10 days). Intersubject variability was particularly large in rabbits and cats and particularly small in Syrian hamsters and sheep; it exceeded intrasubject variability in all species except sheep. A two-way ANOVA of the variability indices identified statistically significant effects of species $(F(15,304)=70.202, p<$ 0.001), type of variability (intrasubject versus intersubject, $F(1,304)=131.213, p<0.001$ ), and interaction of the two factors $(F(15,304)=6.675, p<0.001)$. The effect size, as inferred from $\omega^{2}$, was much greater for the effect of species $\left(\omega^{2}=0.65\right)$ than for the effect of type of variability 
$\left(\omega^{2}=0.08\right)$ or for their interaction $\left(\omega^{2}=0.05\right)$. For all 16 species combined, intrasubject variability averaged $90 \mathrm{~min}$, which was significantly less than the 150-min average of intersubject variability $(t(15)=3.188, p=0.006)$.

\section{Discussion}

The daily activity patterns of the 16 species investigated in this study reflected the diversity of activity patterns described in previous studies of individual species [20, 21]. Some of the patterns that we found were unimodal (such as those of squirrels and hamsters), some were bimodal (such as those of dogs and cats), some were multimodal (such as those of sheep and cows), some were predominantly diurnal (such as those of squirrels and Nile grass rats), some were predominantly nocturnal (such as those of hamsters and mice), and some were almost evenly distributed over the night and day (such as those of rabbits and degus). By standardizing environmental conditions such as photoperiod, ambient temperature, and food availability, as well as the analytical procedures, we were able to increase confidence in the inference that interspecies differences are due mostly to the peculiarities of the circadian systems of the various species. Particularly instructive was the similarity of the activity patterns of the two species of

the genus Mus (house mouse and field mouse). We found the mean activity patterns of these two types of mice to be almost identical (Fig. 1) despite the facts that the two types of mice are distinct species, that M. musculus has been bred in the laboratory for many generations whereas M. booduga was caught in the wild, and that the two species were studied with similar but distinct apparatuses in laboratories located in two different continents.

Analysis of the activity patterns based on onset times and acrophases revealed no significant correlation between these parameters and the body sizes of the various species (Fig. 2). Onset time was more effective than acrophase in separating diurnal species from nocturnal 
species, although the meaning of this difference is not evident. Although dog, sheep, human, horse, and cow exhibited activity onsets shortly after lights-on, their acrophases were late in the day or early in the evening. These five species happen to be the five largest species out of the 16 species studied, and it is possible that larger diurnal mammals generally have later acrophases than smaller diurnal species even though their onset times are not much different from those of the smaller species. Studies of a larger number of species would be necessary to clarify the matter. Later acrophases despite similar onset times could be the result of longer active periods (alpha) or might simply reflect variations in the wave form of the activity rhythms.

Although the robustness of the activity rhythm tended to be greater in smaller species, this trend did not reach statistical significance (Fig. 2). For the body temperature rhythm, a significant increase in robustness was found in larger animals rather than in smaller animals [29], but greater robustness of the temperature rhythm in larger animals could be a simple consequence of thermal inertia and have no implications for the activity rhythm.

Chronotype spreads (the time windows containing 95\% of the individual chronotypes within a species) were found to vary greatly from 40 minutes in sheep to 23 hours in cats (Fig. 3). The chronotype spread previously reported for humans (6 hours) [27] is neither in the short nor in the long end of the continuum. The human chronotype spread is most similar to the chronotype spread of the laboratory rat. A chronotype spread of 6 hours means that most people wake up within a 6-hour window in the morning, with "larks" waking up as early as 6 o'clock and "owls" waking up as late as noon. Although the expression "sheep mentality" usually refers to intellectual conformity, the narrow chronotype spread that we found in sheep indicates that sheep conform to a species-specific activity-onset time situated very close to 90 minutes after sunrise. In contrast, individual cats exhibit great independence from one another and initiate activity each day at times that differ greatly from one individual to another. 
The notion of chronotype spread serves to erode the distinction between diurnal and nocturnal species. It is true that a species with a narrow spread centered at a time after lights-on (such as sheep) can be unequivocally called "diurnal," whereas a species with a narrow spread centered at a time after lights-off (such as the Syrian hamster) can be called "nocturnal."

However, species that have wide chronotype spreads will have some individuals that are mostly active during the day and some that are mostly active during the night, and the proportion of "diurnal" and "nocturnal" individuals will depend on the time of day around which the spread is centered. Dogs and Mongolian gerbils, for example, have about the same chronotype spread, but, because the spread is centered further into the light phase in dogs, every dog could be said to be diurnal, whereas Mongolian gerbils would be 50\% diurnal and 50\% nocturnal. The diurnalnocturnal dichotomy applies only to a limited number of species [30].

Except in sheep (which exhibited very little intersubject variability of onset times), intersubject variability was found to be consistently greater than intrasubject variability (Fig. 4). This means that, in 15 of the 16 species, there was less day-to-day variability in the onset times of individual animals than there was variability between the mean onset times of different individuals. In other words, there was greater consistency in onset times within individuals (mean $\mathrm{SD}=1.5$ hours) than between individuals (mean $\mathrm{SD}=2.5$ hours). Interspecies consistency in onset times (mean $\mathrm{SD}=5.7$ hours) was less than intraspecies consistency.

Although comparisons of intra- and intersubject variabilities in circadian rhythms have rarely been conducted, previous studies of the variability in the free-running periods of various species have found smaller intrasubject variability than intersubject variability [31-34], which is consistent with our findings. Distinguishing the two types of variability has important implications for the management of individual needs, such as in clinical practice in veterinary or human medicine. If intersubject variability is greater than intrasubject variability, then treatments 
designed for an "average" patient may turn out to be too weak (or too strong) for a patient who is not average. On the other hand, if intrasubject variability is greater than intersubject variability, then the notion of an average patient may be useful, but the administration of the treatment to patients may have to be adjusted on a daily or weekly basis. Our finding that intersubject variability of chronotype is larger than intrasubject variability indicates that the concept of an average patient should be avoided in all the studied species except sheep. This discussion is clearly pertinent to human patients, as disruption of the normal phase angle of entrainment (such as that associated with transcontinental travel, with shift work, or even with the extensive use of artificial light in the modern 24-hour society) has been shown to have serious negative health effects, such as cardiovascular disease [35], higher incidence of breast cancer [36], increased occurrence of psychiatric disorders [37], and development of metabolic syndrome [38]. Although the health effects of circadian disruption have not been studied in non-human individuals so far, it is reasonable to expect that similar effects will be found in other mammalian species. As one example, pet dogs and cats are currently experiencing an obesity epidemic similar to that experienced by their human owners in industrialized nations [39].

Although studies of human chronotypes rarely acknowledge it, the classification of individuals along the morningness-eveningness continuum is a reflection of differences in the phase angle of entrainment of the circadian system as it relates to the environmental cycle of light and darkness [2,3]. By analyzing the spread of chronotypes in 16 other mammalian species, the present study provided valuable information to allow placement of the topic of human chronotypes within the broader context of variability in the phase angle of entrainment of circadian rhythms in animals. We showed that chronotype spreads can range from less than an hour in sheep to almost 24 hours in cats, thus extending both below and above the 6-hour spread of human chronotypes. 


\section{Acknowledgments}

Preparation of this article benefitted from intellectual exchanges made possible by a grant from the U.S. National Science Foundation (IOS-1546676). 


\section{References}

1. Horne, J. A., Östberg, O. (1976). A self-assessment questionnaire to determine morningnesseveningness in human circadian rhythms. Int J Chronobiol 4: 97-110.

2. Golombek, D. A., Rosenstein, R. E. (2010). Physiology of circadian entrainment. Physiol Rev 90: 1063-1102.

3. Roenneberg, T., Kumar, C. J., Merrow, M. (2007). The human circadian clock entrains to sun time. Curr Biol 17: R44-R45.

4. Baehr, E. K., Revelle, W., Eastman, C. I. (2000). Individual differences in the phase and amplitude of the human circadian temperature rhythm with an emphasis on morningnesseveningness. J Sleep Res 9: 117-127.

5. Lack, L., Bailey, M., Lovato, N., Wright, H. (2009). Chronotype differences in circadian rhythms of temperature, melatonin, and sleepiness as measured in a modified constant routine protocol. Nat Sci Sleep 1: 1-8.

6. Taillard, J., Sanchez, P., Lemoine, P., Mouret, J. (1990). Heart rate circadian rhythm as a biological marker of desynchronization in major depression: a methodological and preliminary report. Chronobiol Int 7: 305-316.

7. Kerkhof, G. A., Van Dongen, H. P. A., Bobbert, A. C. (1998). Absence of endogenous circadian rhythmicity in blood pressure? Am J Hypertens 11: 373-377.

8. Martin, S. K., Eastman, C. I. (2002). Sleep logs of young adults with self-selected sleep times predict the dim light melatonin onset. Chronobiol Int 19: 695-707.

9. Roemer, H. C., Griefahn, B., Kuenemund, C., Blaszkewicz, M., Gerngroß, H. (2003). The reliability of melatonin synthesis as an indicator of the individual circadian phase position. Milit Med 168: 674-678. 
10. Hasler, B. P., Allen, J. J. B., Sbarra, D. A., Bootzin, R. R., Bernert, R. A. (2010).

Morningness-eveningness and depression: preliminary evidence for the role of BAS and positive affect. Psychiatry Res 176: 166-173.

11. Biss, R. K., Hasher, L. (2012). Happy as a lark: morning-type younger and older adults are higher in positive affect. Emotion 12: 437-441.

12. Ponzi, D., Wilson, M. C., Maestripieri, D. (2014). Eveningness is associated with higher risktaking, independent of sex and personality. Psychol Rep 115: 932-947.

13. Preckel, F., Lipnevich, A. A., Boehme, K., Brandner, L., Georgi, K., Könen, T., Mursin, K., Roberts, R. D. (2013). Morningness-eveningness and educational outcomes: the lark has an advantage over the owl at high school. Br J Educ Psychol 83: 114-134.

14. Jankowski, K. S., Díaz-Morales, J. F., Randler, C. (2014). Chronotype, gender, and time for sex. Chronobiol Int 31: 911-916.

15. Labyak, S. E., Lee, T. M., Goel, N. (1997). Rhythm chronotypes in a diurnal rodent, Octodon degus. Am J Physiol 273: R1058-R1066.

16. Low-Zeddies, S. S., Takahashi, J. S. (2001). Chimera analysis of the Clock mutation in mice shows that complex cellular integration determines circadian behavior. Cell 105: 25-42.

17. Refinetti, R. (2004). Parameters of photic resetting of the circadian system of a diurnal rodent, the Nile grass rat. Acta Sci Vet 32: 1- 7.

18. Masuda, K., Zhdanova, I. V. (2010). Intrinsic activity rhythms in Macaca mulatta: their entrainment to light and melatonin. J Biol Rhythms 25: 361-371.

19. Pfeffer, M., Wicht, H., von Gall, C., Korf, H. W. (2015). Owls and larks in mice. Front Neurol 6: art. 101. 
20. Dunlap, J. C., Loros, J. J., DeCoursey, P. J. (2004). Chronobiology: Biological Timekeeping. Sunderland, Mass.: Sinauer.

21. Refinetti, R. (2016). Circadian Physiology, 3rd Edition. Boca Raton. Fla.: CRC Press.

22. Piccione, G., Caola, G., Refinetti, R. (2005). Daily rhythms of blood pressure, heart rate, and body temperature in fed and fasted male dogs. J Vet Med A 52: 377-381.

23. Ruf, T. (1999). The Lomb-Scargle periodogram in biological rhythm research: analysis of incomplete and unequally spaced time-series. Biol Rhythm Res 30: 178-201.

24. Nelson, W., Tong, Y. L., Lee, J. K., Halberg, F. (1979). Methods for cosinor rhythmometry. Chronobiologia 6: 305-323.

25. Refinetti, R., Cornélissen, G., Halberg, F. (2007). Procedures for numerical analysis of circadian rhythms. Biol Rhythm Res 38: 275-325.

26. Kirk, R. E. (1995). Experimental Design: Procedures for the Behavioral Sciences, 3rd Edition. Pacific Grove, Calif.: Brooks/Cole.

27. Roenneberg, T., Kuehnle, T., Juda, M., Kantermann, T., Allebrandt, K., Gordijn, M., Merrow, M. (2007). Epidemiology of the human circadian clock. Sleep Med Rev 11: 429-438.

28. Sani, M., Refinetti, R., Jean-Louis, G., Pandi-Perumal, S. R., Durazo-Arvizu, R. A., Dugas, L. R., Kafensztok, R., Bovet, P., Forrester, T. E., Lambert, E. V., Plange-Rhule, J., Luke, A. (2015). Daily activity patterns of 2316 men and women from five countries differing in socioeconomic development. Chronobiol Int 32: 650-656.

29. Refinetti, R. (2010). The circadian rhythm of body temperature. Front Biosci 15: 564-594.

30. Refinetti, R. (2008). The diversity of temporal niches in mammals. Biol Rhythm Res 39: 173-192. 
31. DeCoursey, P. (1960). Phase control of activity in a rodent. Cold Spring Harb Symp Quant Biol 25: 49-55.

32. Eskin, A. (1971). Some properties of the system controlling the circadian activity rhythm of sparrows. In: Menaker, M. (Ed.). Biochronometry. Washington, DC: National Academy of Sciences, pp. 55-80.

33. Pohl, H. (1983). Light pulses entrain the circadian activity rhythm of a diurnal rodent (Ammospermophilus leucurus). Comp Biochem Physiol B 76: 723-729.

34. Sharma, V. K. (1996). Light-induced phase response curves of the circadian activity rhythm in individual field mice, Mus booduga. Chronobiol Int 13: 401-409.

35. Nagai, M., Hoshide, S., Kario, K. (2013). Role of 24-hour blood pressure management in preventing kidney disease and stroke. Contrib Nephrol 179: 67-80.

36. Jia, Y., Lu, Y., Wu, K., Lin, Q., Shen, W., Zhu, M., Huang, S., Chen, J. (2013). Does night work increase the risk of breast cancer? A systematic review and meta-analysis of epidemiological studies. Cancer Epidemiol 37: 197-206.

37. Jagannath, A., Peirson, S.N., Foster, R.G. (2013). Sleep and circadian rhythm disruption in neuropsychiatric illness. Curr Opin Neurobiol 23: 888-894.

38. Reiter, R.J., Tan, D.X., Korkmaz, A., Ma, S. (2012). Obesity and metabolic syndrome: association with chronodisruption, sleep deprivation, and melatonin suppression. Ann Med 44: 564-577.

39. Zoran, D.L. (2010). Obesity in dogs and cats: a metabolic and endocrine disorder. Vet Clin North Am Small Anim Pract 40: 221-239. 


\section{Figure Captions}

Fig. 1. Average daily activity rhythms of the 16 species. Each panel was constructed by averaging 10 consecutive days for each animal time-bin by time-bin and then averaging the data for all animals in the same species. The horizontal black and white bars at the top denote the dark and light phases of the prevailing light-dark cycle.

Fig. 2. Means ( \pm SEM) of the onset times (top panel), acrophases (middle panel), and robustness (top panel) of the activity rhythms of various mammalian species as a function of body mass. The horizontal dashed lines indicates the times of lights-on (07:00 hours) and lights-off (19:00 hours). Human data are from Roenneberg and colleagues [27] and Sani and colleagues [28]. The abscissas are in logarithmic scale.

Fig. 3. Chronotype spreads for humans and 16 animal species arranged in ascending order. The chronotype spread for each species is the interval containing $95 \%$ of the individual chronotypes.

Fig. 4. Mean ( \pm SEM) intrasubject and intersubject variabilities of activity onsets of the 16 species in this study. Variability is expressed as the standard deviation of the mean. 
Table 1. The 16 species used in the study.

\begin{tabular}{|c|c|c|c|}
\hline Species & Scientific name & $\begin{array}{l}\text { Body mass } \\
(\mathrm{kg})\end{array}$ & $\begin{array}{l}\text { Sample } \\
\text { size }\end{array}$ \\
\hline Field mouse & Mus booduga & 0.01 & 11 \\
\hline House mouse (C57BL/6) & Mus musculus & 0.03 & 19 \\
\hline Siberian hamster & Phodopus sungorus & 0.05 & 14 \\
\hline Mongolian gerbil & Meriones unguiculatus & 0.06 & 11 \\
\hline Nile grass rat & Arvicanthis niloticus & 0.12 & 18 \\
\hline Palm squirrel & Funambulus pennanti & 0.15 & 10 \\
\hline Syrian hamster & Mesocricetus auratus & 0.16 & 16 \\
\hline Degu & Octodon degus & 0.24 & 8 \\
\hline Laboratory rat (Wistar) & Rattus norvegicus & 0.36 & 16 \\
\hline Fox squirrel & Sciurus niger & 0.8 & 8 \\
\hline Rabbit & Oryctolagus cuniculus & 3 & 16 \\
\hline Cat & Felis catus & 4 & 3 \\
\hline Dog & Canis familiaris & 30 & 6 \\
\hline Sheep & Ovis aries & 40 & 4 \\
\hline Horse & Equus caballus & 550 & 13 \\
\hline Cow & Bos taurus & 700 & 3 \\
\hline
\end{tabular}



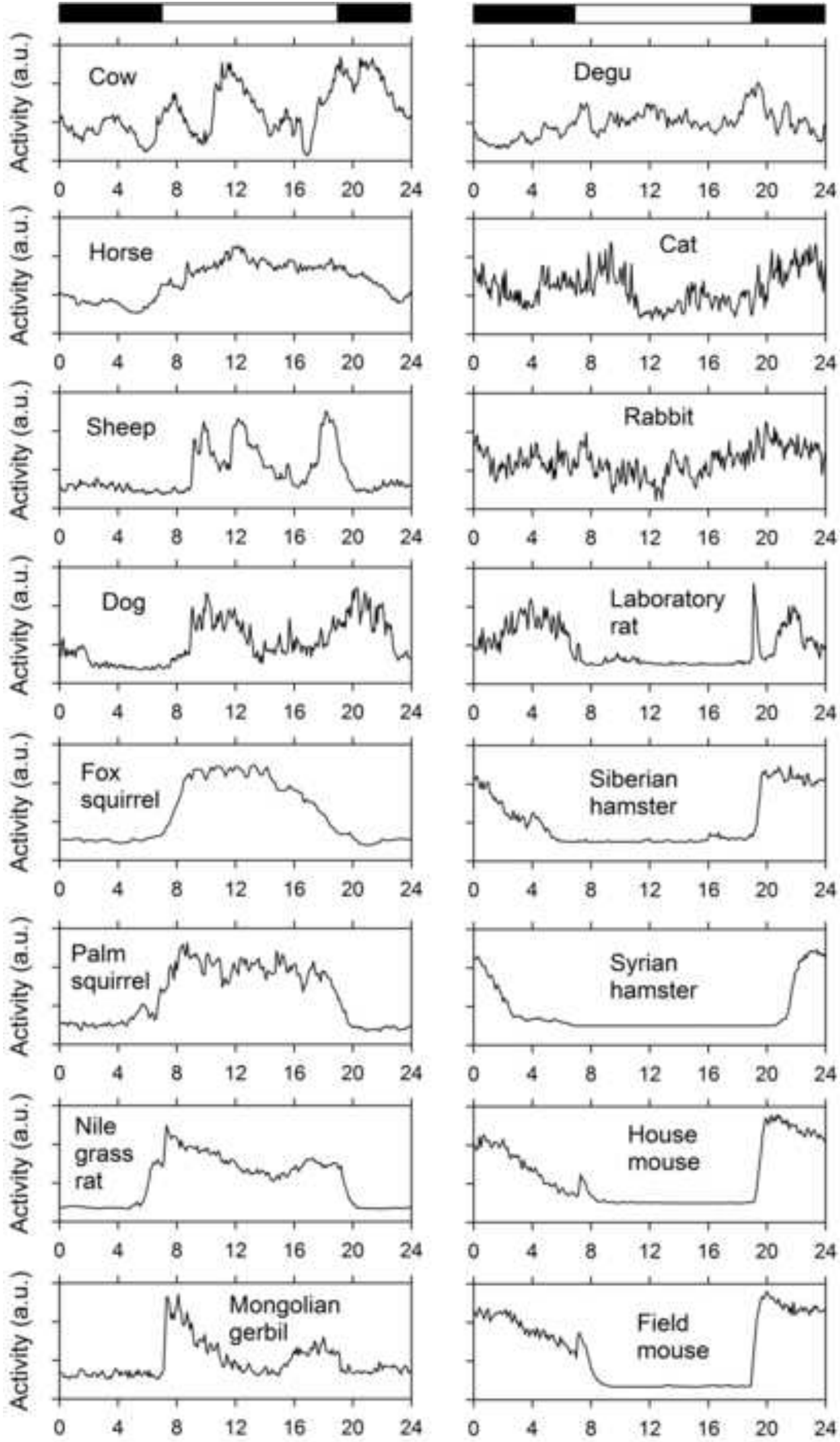

Hours

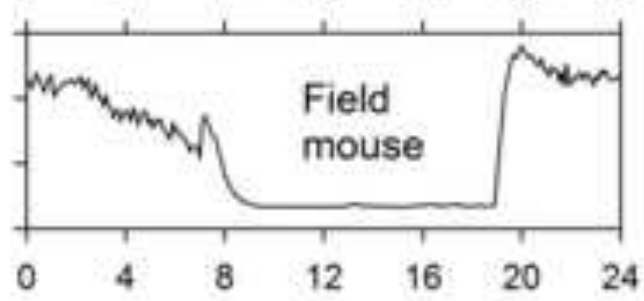

Hours 

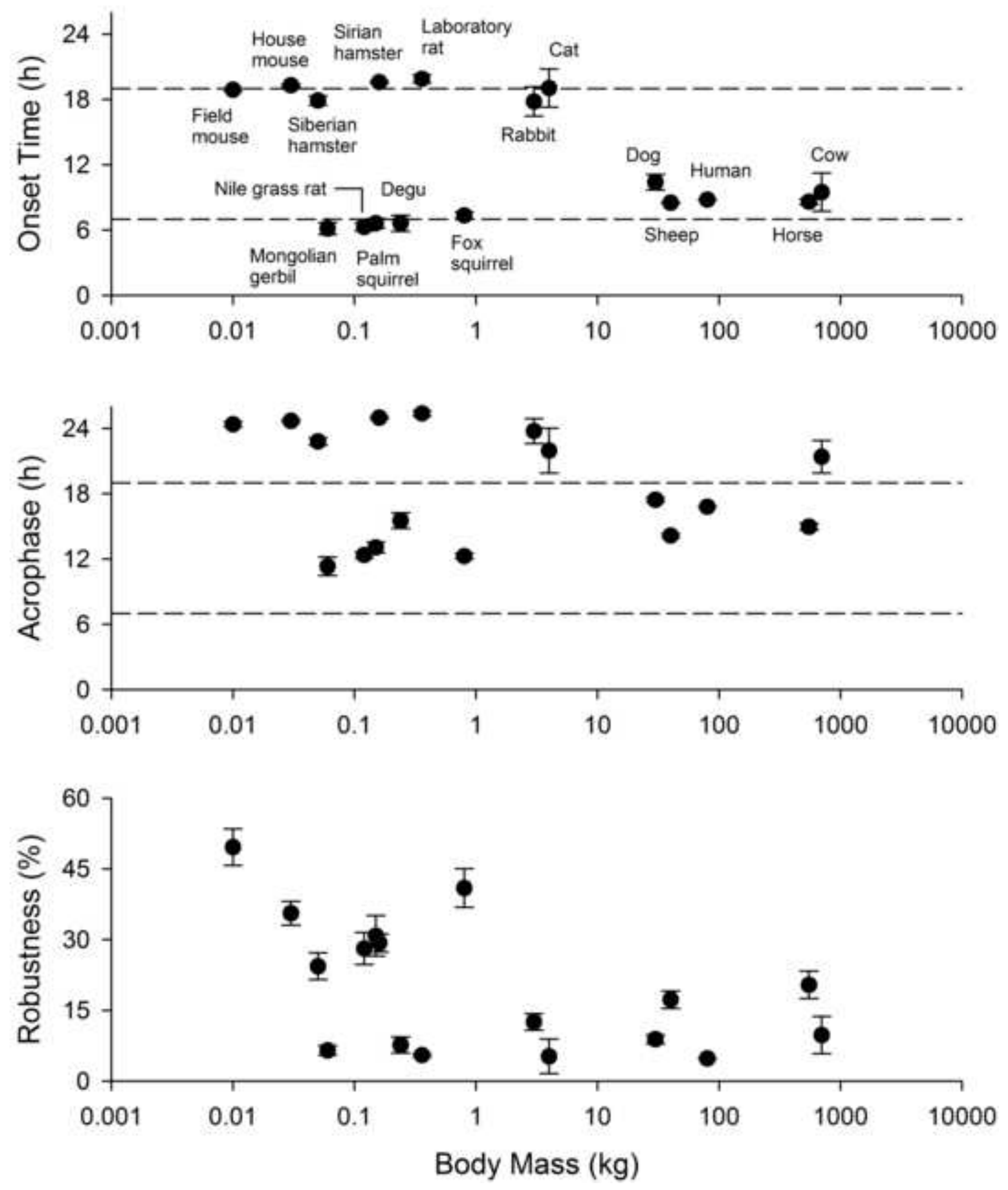

Figure 2 


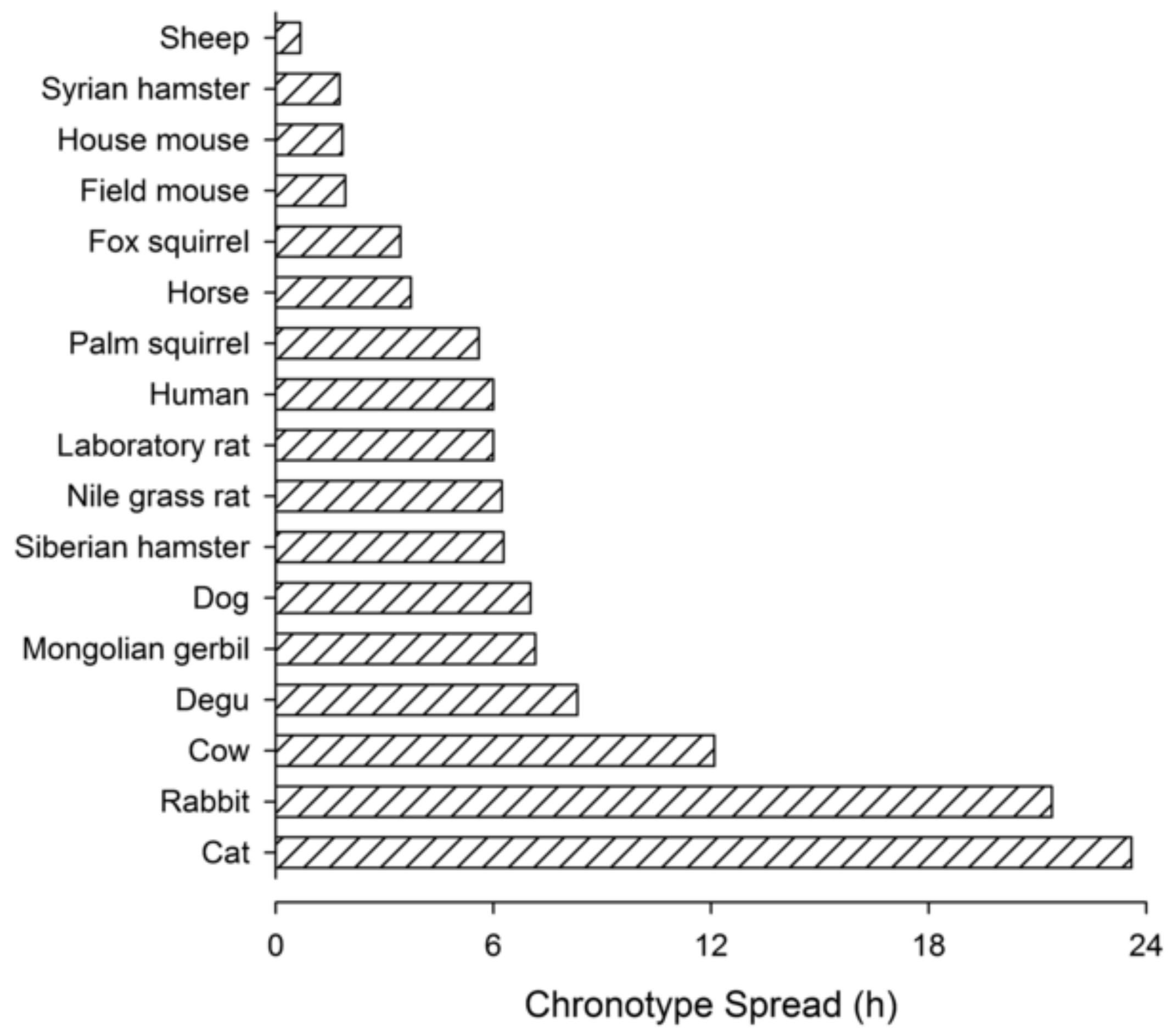




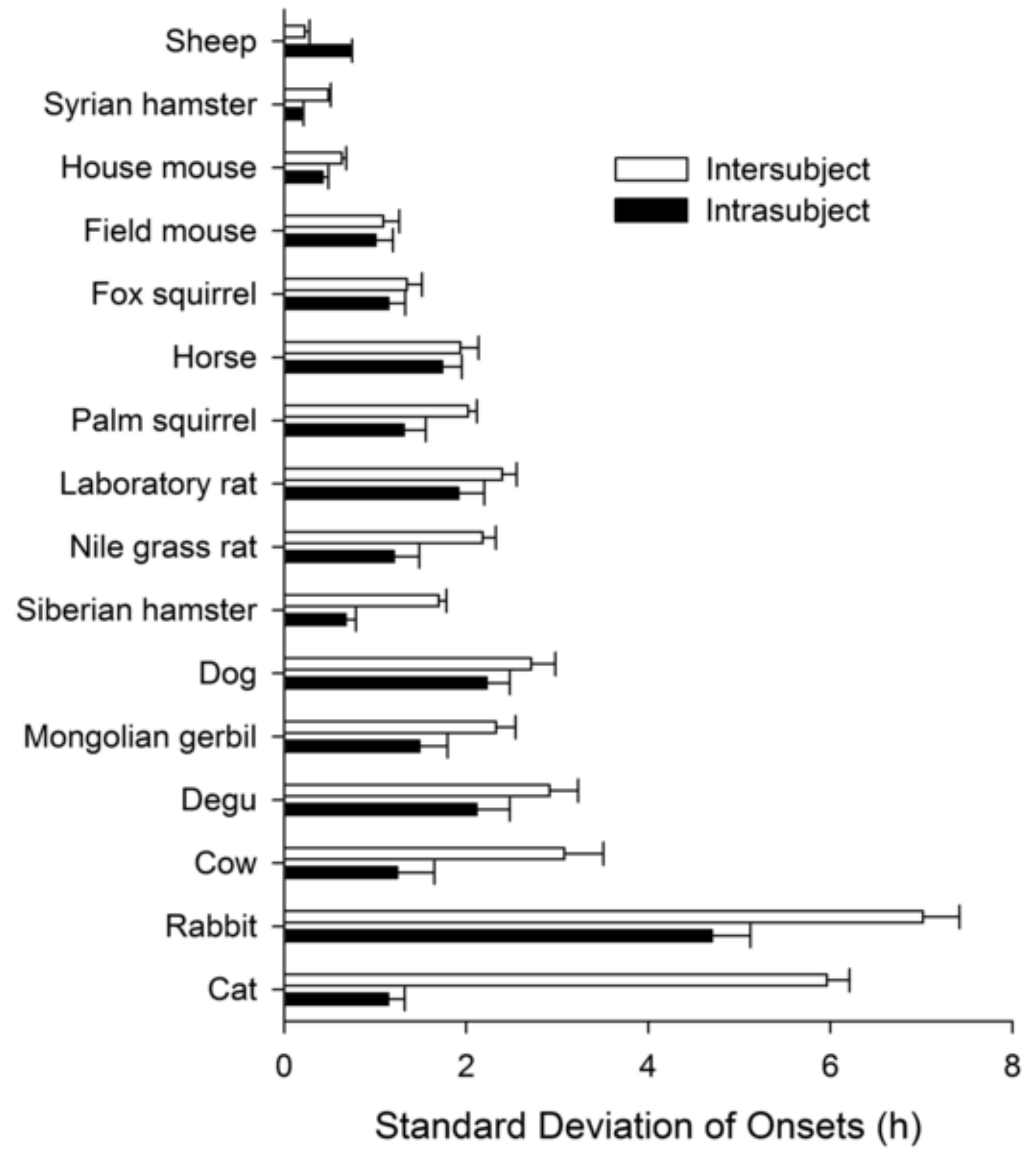

Sheep 르머

Standard Deviation of Onsets (h) 8 